\title{
Human embryonic mesenchymal stem cells participate in differentiation of renal tubular cells in newborn mice
}

\author{
LI YUAN $^{1 *}$, HOU-QI LIU ${ }^{2}$ and MIN-JUAN WU ${ }^{2 *}$ \\ ${ }^{1}$ Department of Nephrology, Affiliated Hospital of Nantong University, Nantong, Jiangsu 226001; \\ ${ }^{2}$ Department of Histology and Embryology, Research Center of Developmental Biology, \\ Second Military Medical University, Shanghai 200433, P.R. China
}

Received March 24, 2015; Accepted April 25, 2016

DOI: $10.3892 /$ etm.2016.3383

\begin{abstract}
Stem cells are used with increasing success in the treatment of renal tubular injury. However, whether mesenchymal stem cells (MSC) differentiate into renal tubular epithelial cells remains controversial. The aims of the present study were to observe the localization of human embryonic MSCs (hMSCs) in the kidneys of newborn mice, and to investigate hMSC differentiation into tubular epithelium. Primary culture hMSCs were derived from 4-7-week-old embryos and labeled with the cell membrane fluorescent dye PKH-26. The degree of apoptosis, cell growth, differentiation and localization of hMSCs with and without this label were then determined using immunohistochemical methods and flow cytometry. hMSCs and PKH26-labeled hMSCs were revealed to differentiate into chondrocytes and adipocytes, and were demonstrated to have similar proliferative capability. In the two cell types, the antigens CD34 and CD45, indicative of hematopoietic lineages, were not expressed; however, the expression of the mesenchymal markers CD29 and CD90 in MSCs, was significantly increased. During a 4-week culture period, laser confocal microscopy revealed that PKH26-labeled hMSCs in the kidneys of newborn mice gradually dispersed. Two weeks after the injection of the PKH26-labeled cells, the percentage of PKH26-labeled hMSCs localized to the renal tubules was $10 \pm 2.1 \%$. In conclusion, PKH26 labeling has no effect on
\end{abstract}

Correspondence to: Dr Li Yuan, Department of Nephrology, Affiliated Hospital of Nantong University, 20 Xisi Road, Nantong, Jiangsu 226001, P.R. China

E-mail: yuanlint@163.com

Dr Min-Juan Wu, Department of Histology and Embryology, Research Center of Developmental Biology, Second Military Medical University, 800 Xiangyin Road, Shanghai 200433, P.R. China

E-mail:minjuanwu@qq.com

*Contributed equally

Key words: mesenchymal stem cells, embryo, PKH-26, acute kidney injury, renal tubular epithelium
hMSC differentiation, proliferation and mesenchymal cell surface features, and hMSCs injected into the kidneys of newborn mice may transform to renal tubule epithelium.

\section{Introduction}

Mesenchymal stem cell (MSC) research is a key topic in current stem cell research. Multiple previous studies have demonstrated that MSCs have therapeutic effects on acute kidney injury (AKI), immunoglobulin A nephropathy and other renal diseases (1-3). AKI is recognized as a common disease which is expensive to manage, prolongs hospitalization and is associated with increased mortality. The mortality rate for AKI has been reported at $24 \%$ in hospital patients, and increases with the severity of AKI (4). Clinically, novel and more effective strategies for the treatment of AKI are expected. MSCs have been applied and provided satisfactory therapeutic effects for AKI in animals and humans $(2,3,5)$; there are multiple mechanisms involved in the therapeutic actions of MSC in AKI treatment (1).

The hypothesis that MSCs may transform to renal tubular epithelial cells remains controversial. The discrepancy in results is likely to be associated with the types of stem cell, disease models and labeling and detection methods used $(1,5,6)$. The first MSCs, and the most widely used, are bone marrow-derived MSCs (BMSCs) (6). However, other sources of MSCs have also emerged in clinical and research settings of renal regenerative therapy and chronic kidney disease, including adult and fetal tissue and umbilical cord blood $(7,8)$. However, previous studies on MSCs sourced from human early embryos are limited. Compared with MSCs derived from adult tissues, a number of features of embryonic stem cells differ, including biological activities, such as cytokine expression and cell adhesion molecules $(9,10)$, differentiation potential, in vivo migration and proliferation of embryo stem cells are markedly increased compared with adult stem cells, and the immunogenicity of embryonic stem cells is reduced (11-15). Embryo-derived MSCs can be frozen in the laboratory and amplified immediately to meet the requirement for treatment. However, alternative sources of embryonic stem cells would be beneficial for research and therapeutic purposes (16). Previous studies on MSCs sourced from human early embryos are limited. 
The current study hypothesized that MSCs derived from human early embryos have an improved capability to differentiate into tubular cells. The aim of the present study was therefore to determine whether human embryonic MSCs (hMSCs) are able to transform into renal tubular cells in the kidneys of newborn mice.

\section{Materials and methods}

Cell culture and labeling with PKH26. hMSCs were obtained from human embryos aged 4-7 weeks old, provided by $\mathrm{Dr}$ Minjuan Wu (Research Center of Developmental Biology and Department of Histology and Embryology, Second Military Medical University, Shanghai, China). The human embryos were obtained from voluntary terminations of pregnancy with RU486 anti-progesterone compound (17). The Committee on Ethics of Biomedicine Research (Second Military Medical University, Shanghai, China) reviewed and approved all human research protocols, and all donors provided written informed consent. The hMSCs were grown as described previously (8) and stored at the Department of Histology and Embryology of the Second Military Medical University (Shanghai, China). The hMSCs were maintained in Dulbecco's minimal essential medium (DMEM; Invitrogen; Thermo Fisher Scientific, Inc., Waltham, MA, USA) supplemented with $10 \%$ fetal calf serum at $37^{\circ} \mathrm{C}$ in an atmosphere of $5 \%$ carbon dioxide.

As a type of lipophilic dye and emission of red fluorescence, PKH26 can be combined with cell membranes irreversibly and conduct fluorescence labeling for numerous types of cells. hMSCs at passage 4 were labeled with the red fluorescent dye PKH26 (Sigma-Aldrich, St. Louis, MO, USA) according to the manufacturer's protocol. Briefly, the $80-90 \%$ confluence hMSCs were trypsinized by $0.25 \%$ Trypsin solutions (Invitrogen; Thermo Fisher Scientific, Inc.), washed using serum-free DMEM and resuspended in $1 \mathrm{ml}$ of Diluent $\mathrm{C}$ from the PKH26 Red Fluorescent Cell Linker kit (cat. no. PKH26-GL; Sigma-Aldrich). The cell suspension was mixed with an equal volume of the labeling solution (containing $4 \mathrm{nM}$ PKH26; final concentration, $4 \mathrm{nM} \mathrm{PKH} 26$ ) and incubated at $25^{\circ} \mathrm{C}$ for $5 \mathrm{~min}$. The staining reaction was stopped by the addition of $2 \mathrm{ml}$ fetal bovine serum, cells were washed 3 times with DMEM and observed using epifluorescence microscopy.

In vitro counting. PKH26-positive hMSCs were observed by fluorescence microscopy at five different time points following addition of the PKH-26 label (24 h, 1 week, 2 weeks, 3 weeks and 4 weeks). A total of 5 fields of view (magnification, $x 400$ ) were selected for every time point. Red fluorescent cells were counted in each field of view, and the labeling rate was calculated as: The number of PKH26-positive cells/total number of cells.

Proliferation. Cell growth curves were drawn to compare the proliferation between hMSCs and PKH26-labeled hMSCs. The cells were grown on 24-well plates at a density of 100 cells $/ \mathrm{cm}^{2}$. Every $24 \mathrm{~h}$, the cell number in 4 randomly-selected wells was counted, and mean values were calculated using a hemocytometer counting chamber. Cell growth curves of hMSCs and PKH26-labeled hMSCs from days 1-7 were then calculated from these values, as a function of incubation time.
Fluorescence activated cell sorting (FACS) analysis. Surface markers of the hMSCs were analyzed by FACS. The following monoclonal antibodies were used: Fluorescein isothiocyanate (FITC)-conjugated anti-CD90 (cat. no. 328108), anti-CD34 (cat. no. 343604) and anti-CD45 (cat. no. 368508) and phycoerythrin-conjugated anti-CD29 (cat. no. 303004) (Biolegend). The analysis was performed by a FACSCalibur cytometer (BD Biosciences, Franklin Lakes, NJ, USA). hMSCs were stained with antibody (1:100) and incubated at $4^{\circ} \mathrm{C}$ for $30 \mathrm{~min}$. At least 10 cell samples were acquired for each analysis.

Apoptosis was assessed by FITC-annexin V and propidium iodide staining (Annexin V-FITC Apoptosis Detection kit, Bipec Biopharma, Cambridge, MA, USA) according to the manufacturer's instructions. Cells were analyzed by FACS at 594-nm excitation (green fluorescence) for annexin-stained cells, and excitation (red) for cells stained with propidium iodide.

Chondrogenic and adipogenic differentiation. The hMSCs and PKH26-hMSCs were seeded onto 6-well plates, and differentiation of these cells into chondrocytes and adipocytes was induced at $40-50 \%$ confluence. To induce chondrocyte differentiation, cells were cultured with chondrogenic differentiation medium comprising $1.5 \times 10^{-4} \mathrm{mg} / \mathrm{ml}$ ascorbic acid and $1 \mathrm{ng} / \mathrm{ml}$ human recombinant transforming growth factor- $\beta$ (Sigma-Aldrich). Immunohistochemistry was performed to examine the presence of types II collagen. Cell-seeded constructs were rinsed with $1 \mathrm{X}$ phosphate-buffered saline, fixed in $4 \%$ formalin for $24 \mathrm{~h}$ and embedded in paraffin. Slides were incubated with $10 \%$ normal goat serum to block non-specific sites and mouse monoclonal collagen type II (1:100; 1 mg/ml; cat. no. ab3092; Abcam, Cambridge, UK) primary antibodies were applied for $1 \mathrm{~h}$ at $22^{\circ} \mathrm{C}$. Secondary antibody (anti-mouse immunoglobulin $\mathrm{G}$ biotin conjugate; 1:200; $2.1 \mathrm{mg} / \mathrm{ml}$; cat. no. B7151; Sigma-Aldrich) was added for $1 \mathrm{~h}$ followed by incubation with ABC reagent (Vectastain PK-400; Vector Laboratories, Inc., Peterborough, UK) for $45 \mathrm{~min}$. To induce adipocyte differentiation, cells were cultured with adipogenic differentiation medium comprising $1 \times 10^{-8} \mathrm{~mol} / 1$ dexamethasone and $1 \times 10^{-10} \mathrm{~mol} / 1$ insulin (Sigma-Aldrich) (8). Two weeks after differentiation, adipocytes were identified by the existence of lipid vesicles, using staining with Oil Red O (Sigma-Aldrich).

In vivo experiments. This study conformed to the Guide for the Care and Use of Laboratory Animals (18). Pregnant Kunming mice were purchased from the Shanghai Laboratory Experimental Animal Center of the Second Military Medical University (Shanghai, China). Ethical approval was provided by the Ethics Committee of Biomedicine Research (Second Military Medical University). All mice were allowed free access to standard laboratory chow and tap water, housed in a room with constant temperature $\left(22^{\circ} \mathrm{C}\right)$ and a $12 / 12-\mathrm{h}$ light/dark cycle.

Kunming mice ( $\mathrm{n}=6$; weight, $2.01 \pm 0.18 \mathrm{~g}$ ) were narcotized by ether for $2 \mathrm{~min}, 2$ days post-birth for $2 \mathrm{~min}$. A microsyringe was used to slowly inject $20 \mu 1$ cell suspension in phosphate-buffered saline containing $4 \times 10^{3}$ PKH26-hMSCs into the left kidney at a constant rate. The uninjected right kidney served as a control. 
A

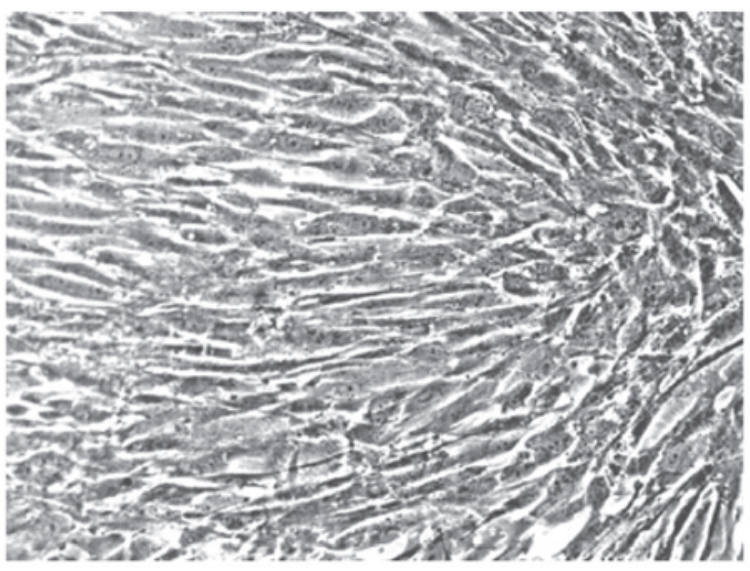

B

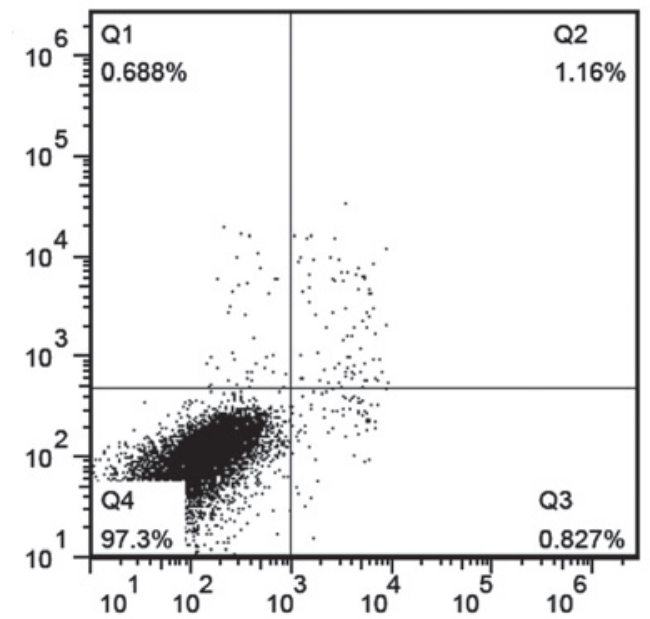

Figure 1. hMSC morphology and apoptosis. (A) Cells present with a fibroblast-like morphology. Magnification, x400; (B) fewer than 1\% cells are undergoing apoptosis. hMSCs, human embryonic mesenchymal stem cells.

A
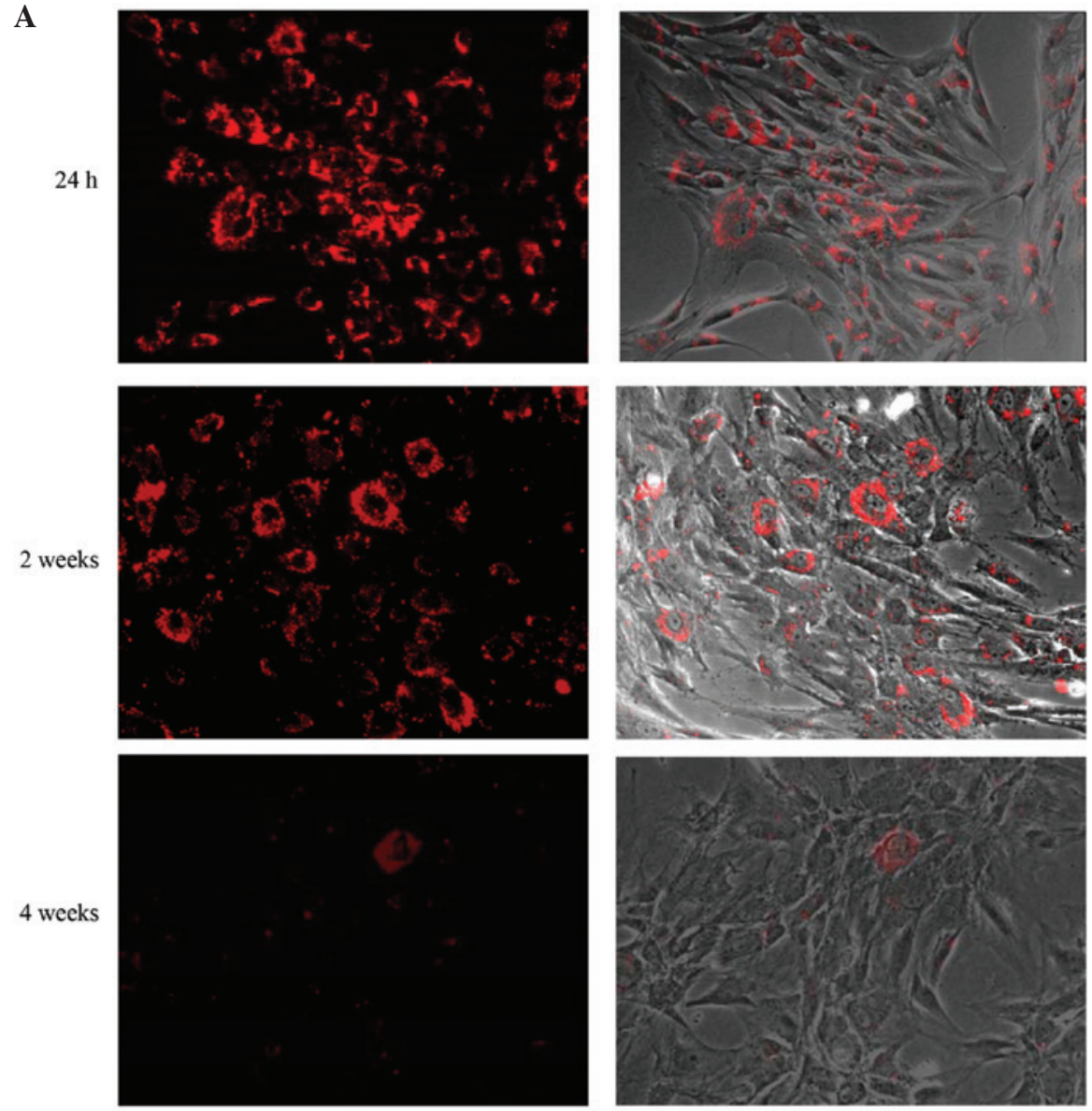

B

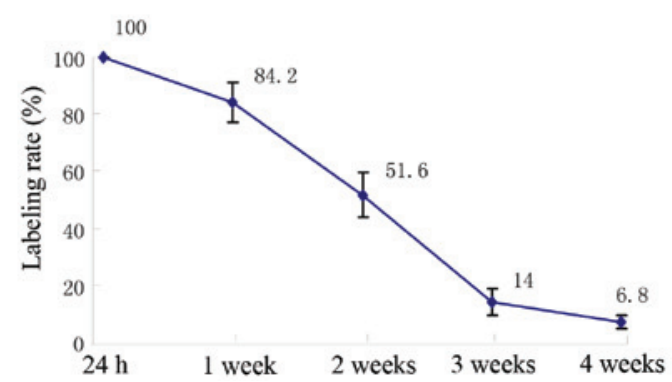

Figure 2. Determination of PKH26 labeling of hMSCs. (A) hMSCs labeled with PKH26 were observed by fluorescence microscopy; (B) the labeling rate at different time points subsequent to PKH26 labeling. hMSCs, human embryonic mesenchymal stem cells. 


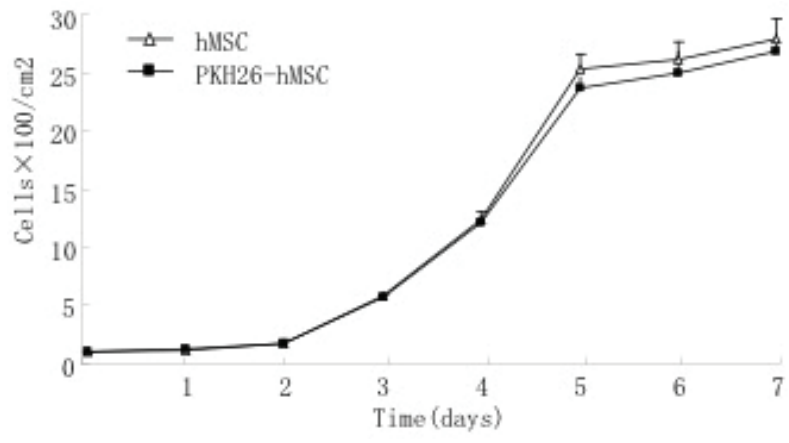

Figure 3. Characterization of PKH26-labeled hMSCs. PKH26 labeling had no significant effect on hMSC cell growth compared with unlabeled hMSCs. hMSCs, human embryonic mesenchymal stem cells.

Renal morphology. Within 4 weeks of PKH26-hMSC transplantation, frozen sections were extracted from kidney tissues weekly. Successive frozen sections (thickness, $5 \mu \mathrm{m}$ ) were made following Optimal Cutting Temperature (OCT) compound embedding. Subsequent to drying for $30 \mathrm{~min}$ at room temperature, the sections were fixed with cold acetone for $10 \mathrm{~min}$. An Olympus IX70 fluorescence microscope (Olympus Corporation, Tokyo, Japan) and a laser scanning confocal microscope (Leica Microsystems, Inc., Buffalo Grove, IL, USA) were used to observe and analyze the sections.

The localization of PKH26-labeled hMSCs in the kidneys was observed 2 weeks after the injection of cells. Samples were frozen immediately in liquid nitrogen, embedded in OCT compound, sliced to $5 \mu \mathrm{m}$ sections, fixed in acetone for $10 \mathrm{~min}$, and incubated for $30 \mathrm{~min}$ at $22^{\circ} \mathrm{C}$ with FITC-labeled wheat germ agglutinin (WGA; Vector Laboratories, Inc.). Nuclei were stained with 4,6-diamidino-2-phenylindole dihydrochloride (Sigma-Aldrich). PKH26-positive cells were counted in 10 frozen renal sections per mouse ( $n=3$ mice). The number of stem cells in kidney tubules was also counted.

Statistical analysis. Stata version 14.0 (StataCorp LP, College Station, TX, USA) was used to perform all statistical analyses. Data are presented as the mean \pm standard error of the mean, and $\mathrm{P}<0.05$ was considered to represent a statistically significant difference. For comparison between unpaired groups, Student's t-test was employed.

\section{Results}

Morphological observation and apoptosis of hMSCs. In primary culture, cell adherence occurred within $12 \mathrm{~h}$ after plating and culture medium was changed after $24 \mathrm{~h}$. Cellular morphology was different to the primary culture. All the stem cells were fibroblast-like at passages 4-5 (Fig. 1A). Fewer than $1 \%$ of cells at passage 5 were undergoing apoptosis when analyzed by flow cytometry (Fig. 1B).

In vitro observation of hMSCs following labeling with PKH26. PKH26 was evenly distributed on the hMSC cell membrane $24 \mathrm{~h}$ after PKH26 labeling, presenting as red fluorescence under microscopy (Fig. 2A). The PKH26 labeling rate was $98.6 \pm 1.83 \%$ (Fig. 2B). With increased cell culture time, fluorescence uniformity on the cell membranes decreased,
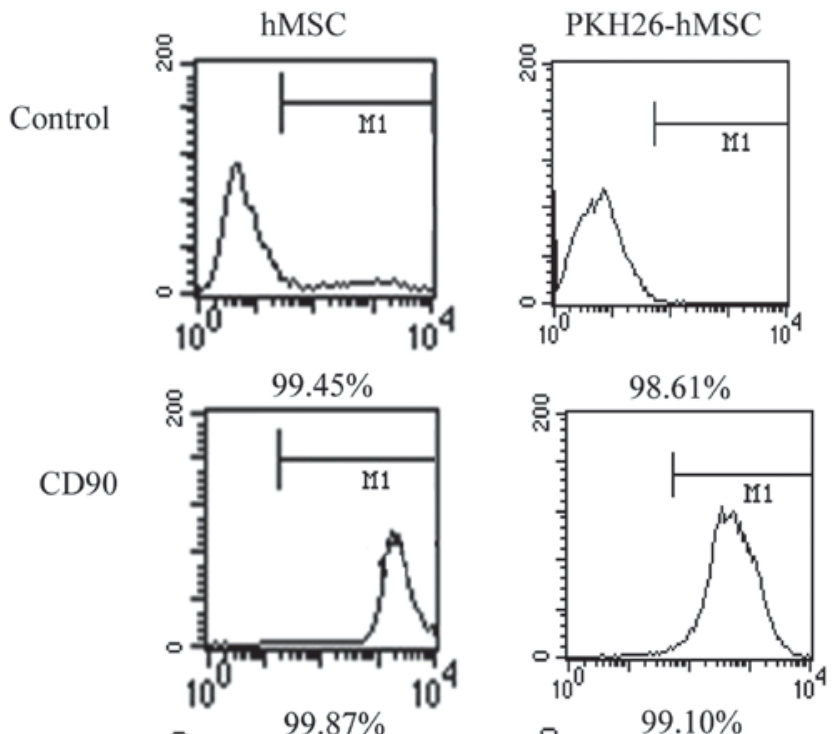

CD29
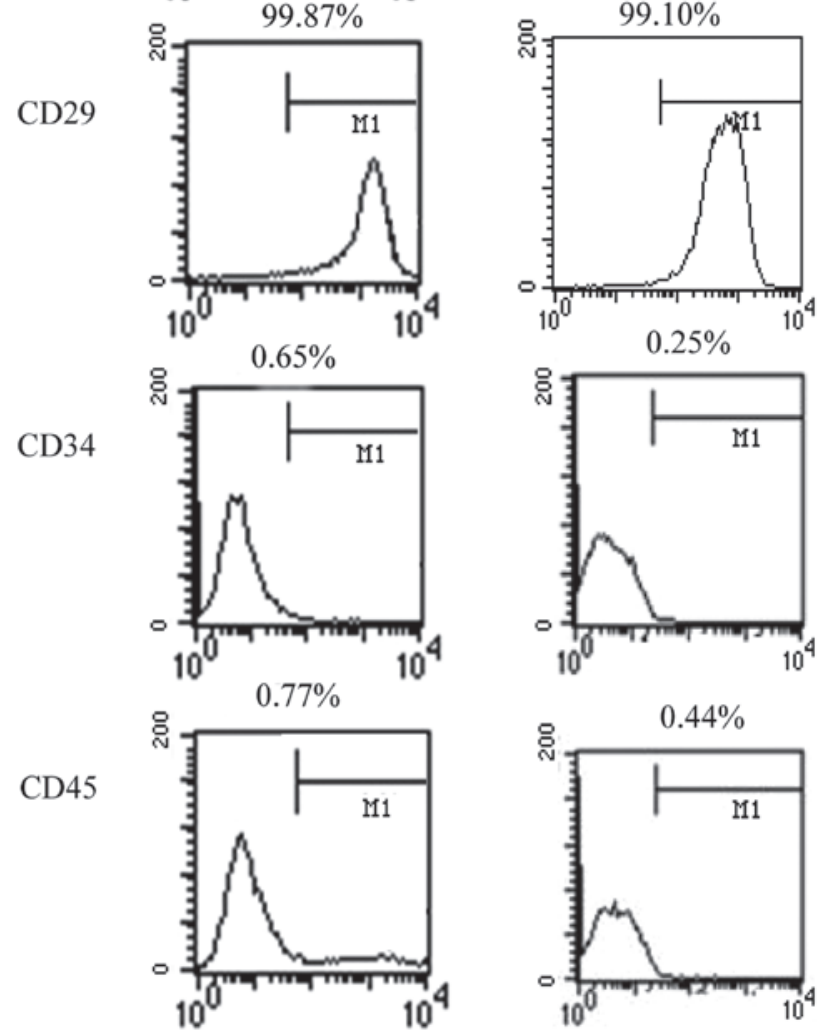

Figure 4. Differentiation of hMSCs and PKH26-labeled hMSCs, determined by flow cytometric analysis, revealing positive staining for the mesenchymal markers CD90 and CD29, and negative staining for the hematopoietic lineage markers CD34 and CD45 ( $\mathrm{n}=3$ repeats per cell line). hMSCs, human embryonic mesenchymal stem cells.

the intensity of labeling progressively weakened, and the labeling rate continuously declined (Fig. 2). Two weeks after PKH26 labeling, the red fluorescence was apparent in half of the hMSCs. Four weeks after labeling, the red fluorescence remained in $\sim 7 \%$ of hMSCs (Fig. 2).

PKH26 labeling has no effect on hMSC proliferation. No statistically significant difference was observed between the growth curves of hMSCs and PKH26-hMSCs $(\mathrm{P}<0.05)$, which indicated that PKH26 label had no marked effect on hMSC proliferation (Fig. 3). 


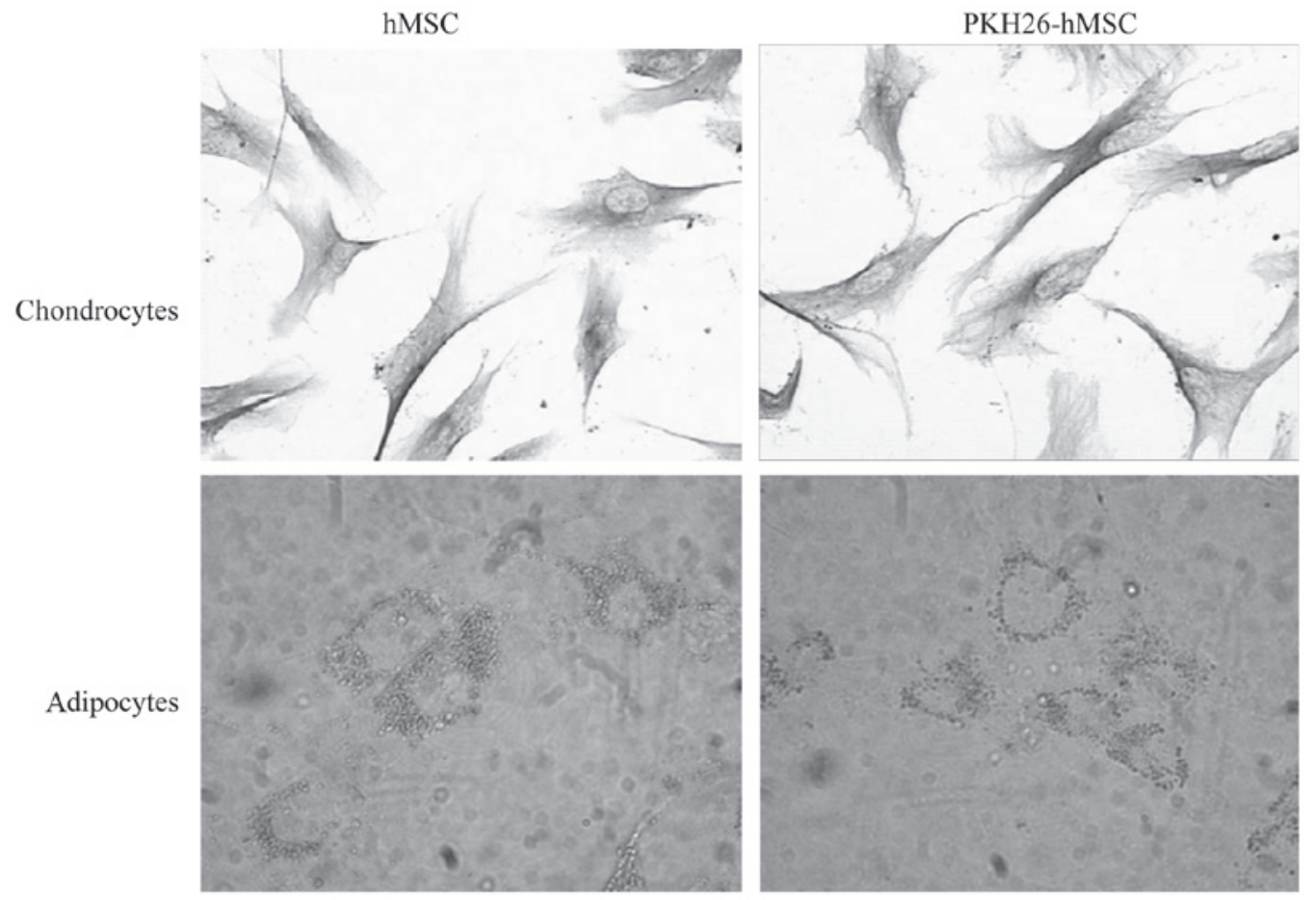

Figure 5. Differentiation of hMSCs and PKH26-labeled hMSCs, revealing the multipotency of these cells as differentiation into chondrocytes and adipocytes, as demonstrated by type II collagen immunostaining and Oil Red O staining, respectively ( $\mathrm{n}=3$ repeats per cell line). Magnification, $\mathrm{x} 400$.

PKH26 labeling does not affect the expression of stem cell markers on hMSCs. Flow cytometric analysis revealed that hMSCs and PKH26-hMSCs were positive for the mesenchymal markers CD29 and CD90, but negative for the markers of hematopoietic lineages CD34 and CD45 (Fig. 4).

PKH26 labeling does not affect the differentiation of hMSCs. Following adipocyte induction, the intracellular lipid droplets continuously enlarged and fused. Subsequent to chondroblast induction, numerous cells had irregular and polygonal morphology, the cell volume increased and contained many particles. The cells contained numerous type II collagen positive brown particles in the cytoplasm, identified by immunohistochemical staining. hMSCs and PKH26-hMSCs were differentially induced into chondrocytes and adipocytes, as demonstrated by Oil Red O staining and type II collagen immunostaining, respectively $(n=3$; 30 cells per repeat; Fig. 5).

hMSCs may differentiate into renal tubular epithelium. The PKH26-labeled fluorescent cells were observed to assemble around the injection site $24 \mathrm{~h}$ after the injection. However, two weeks later, the cells had migrated along the renal tubule, and the red fluorescent cells were apparent in most tissue sections. Four weeks following the injection, fluorescence remained dispersed throughout the kidneys, although the intensity of the fluorescence had weakened (Fig. 6A). FITC-labeled WGA was used to visualize the glycoprotein and sialic acid of cell membranes, and was used to label the renal tubules in the present study. Using confocal microscopy, co-localization of FITC-WGA and PKH26-hMSCs were observed, demonstrating these cells in the kidney tubules. Two weeks after the cell injection, $10 \pm 2.1 \%$ of
PKH26-labeled hMSCs were demonstrated to be localized to the renal tubules, as determined by laser scanning confocal microscopy (Fig. 6B).

\section{Discussion}

MSCs are currently used to research organ development and disease treatment, and these are predominantly derived from adult bone marrow. It has previously been suggested that MSCs have multiple roles, which have been investigated in the treatment of numerous diseases (19); however, the key limitation of using MSCs in such treatments is the low cell quantity available (20). The number of MSCs in adult bone marrow is extremely low, ranging between 0.01 and $0.00001 \%$ of human bone marrow mononuclear cells, according to different donors $(21,22)$. Furthermore, the quantity and differentiation of MSCs in bone marrow reduce with increasing age (23). Compared with adult MSCs, fetal MSC have a high proliferative capability: In identical in vitro culture conditions, the population doubling time is $32.3 \pm 2.5 \mathrm{~h}$ in fetal MSCs and 116.6 $\pm 22.4 \mathrm{~h}$ in adult MSCs (24). In culture, the number of fetal MSCs can be increased several million fold without the loss of phenotype such as CD19, CD44, Cd166, Cd103, SH3 and SH4 (11). Adult MSCs express a moderate amount of human leukocyte antigen (HLA)I and HLAII, while fetal MSCs express extremely low or no HLAI and do not express HLAII, meaning that the immunogenicity of fetal MSCs is much weaker (14). Adult stem cell treatment may cause hyperplasia of the lymphatic system, which is not induced by fetal MSCs. Fetal MSCs and BMSCs can inhibit adult peripheral blood lymphocyte (PBL) proliferation induced by numerous mitogensm such as concanavalin $\mathrm{A}$ (ConA), phytohaemagglutinin (PHA), Staphylococcus aureus ( $\mathrm{SpA}$ ) and poke weed mitogen (PWM) $(11,15)$. Therefore, fetal MSC treatment 
A
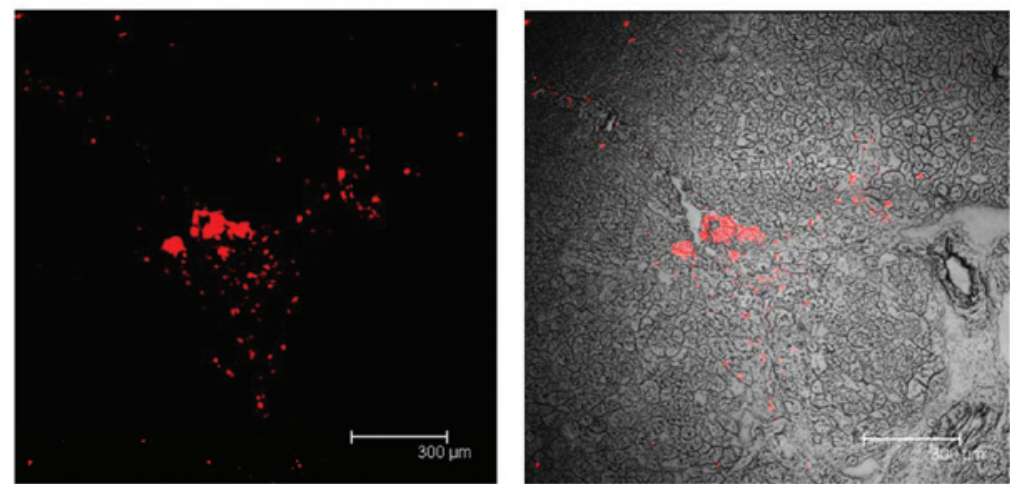

2 weeks
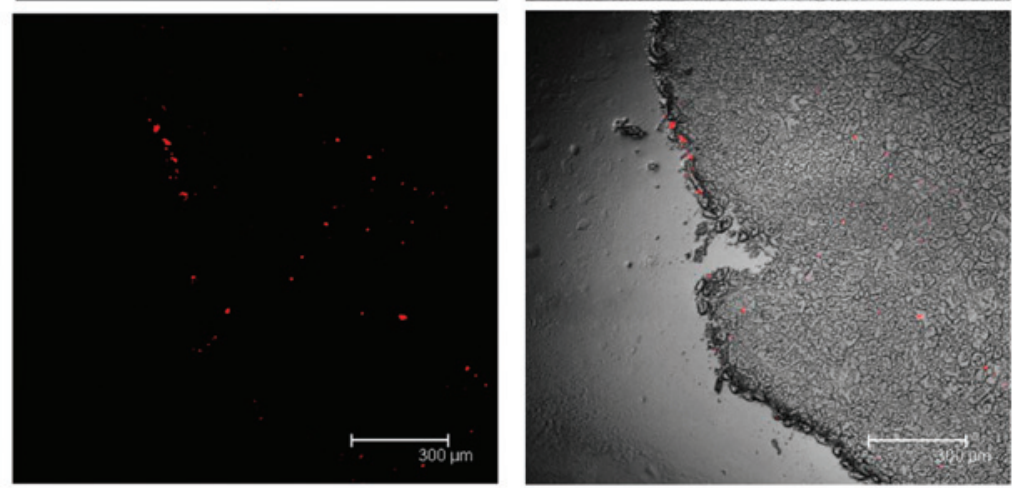

B
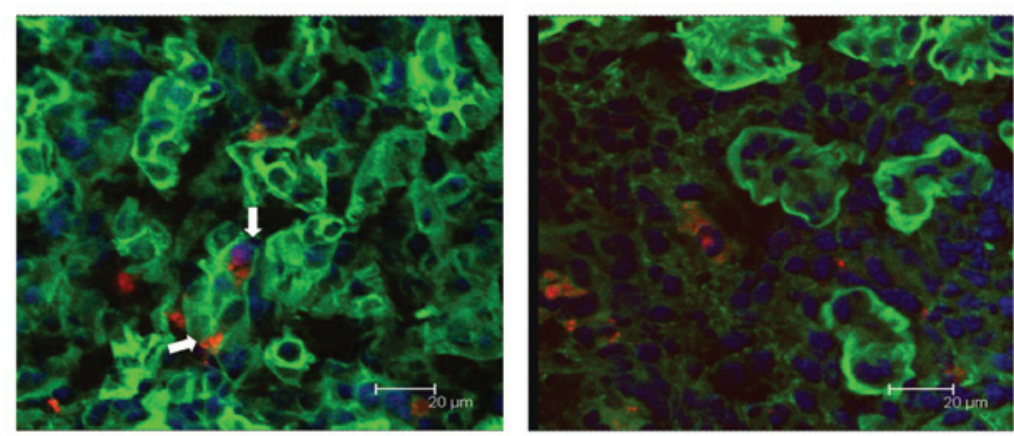

Figure 6. PKH26-labeled hMSC dispersal in mouse kidneys. (A) PKH26-labeled hMSC distribution in the kidney, as observed under confocal microscopy, 2 and 4 weeks after hMSC injection. Magnification, x10. Scale bar=300 $\mu \mathrm{m}$; (B) representative micrographs of kidney tissue after 2 weeks from newborn mice injected with PKH-26-labeled hMSCs. White arrows/red fluorescence indicate hMSCs, localized to renal tubules. hMSCs, human embryonic mesenchymal stem cells. Scale bar, $20 \mu \mathrm{m}$.

has some unique advantages, such as an improved proliferative capacity, phenotype stability and low immunogenicity.

PKH26, a lipophilic emitter of red fluorescence, may be irreversibly bound to the cell membrane to enable fluorescent labeling of multiple cell types (25).Importantly, under appropriate labeling conditions, cell activity is not affected: PKH26 labeling does not affect cell proliferation and the expression of adhesion molecules (26,27). PKH26 is therefore an ideal tool to study cell migration and interactions in vivo and in vitro that is frequently used in cellular tracking studies (25). Shao-Fang et al (28) studied the phenotype, differentiation, proliferation, cell cycle and apoptosis of human umbilical mesenchymal stromal cells following PKH26 labeling, reporting that no effect on the above indexes was observed. In the present study, it was also demonstrated that PKH26 had no significant effect on cell morphology and proliferation. FACS results demonstrated that neither hMSCs nor PKH26-labeled hMSCs expressed hematopoietic lineage markers but did express mesenchymal cell markers. hMSCs and PKH26-labeled hMSCs were also able to differentiate into chondrocytes and adipocytes upon induction. Together, these analyses suggested that PKH26 labeling did not alter the MSC characteristics in hMSCs, indicating that PKH26 labeling is an effective way to label live hMSCs.

The limitation of PKH26 labeling is the gradual attenuation of fluorescence, occurring due to absence of sufficient fluorescent molecules as the cells divide $(25,27,29)$. Ude et al (27) used PKH26 to label MSCs and adipose-derived stem cells, revealing that the red fluorescence remained when the cells were subcultured in vitro to passage 6 at the 49th day, but that the fluorescence intensity markedly weakened. In the present study, fluorescence intensity and the number of PKH26-labeled hMSCs were reduced with time; four weeks after labeling, only $7 \%$ of labeled cells were observed. Based on the present results, the renal localization of PKH26-hMSC was also observed at 2 weeks after the injection.

However, recent studies have demonstrated that BMSCs from mice and humans may cross lineage boundaries and form functional components of other tissues, expressing 
tissue-specific proteins in organs such as the heart, liver, brain, skeletal muscle and vascular endothelium (30-32). Numerous experiments have also reported that stem cells have a therapeutic effect when treating glomerular and tubular diseases $(1,6)$. In previous studies aiming to treat tubular damage with stem cells, AKI has been the most widely used disease model; however, the exact therapeutic mechanism of stem cells in AKI remains under intensive investigation. For instance, a previous study demonstrated that stem cells exert their therapeutic effects in AKI through a paracrine/endocrine mechanism, and that MSCs produce a variety of cytokines and growth factors $(33,34)$. Other studies revealed that microvesicles derived from human MSCs may protect the kidneys from toxic injury through horizontal transfer of mRNA, RNA-dependent apoptosis resistance and in vitro proliferation $(35,36)$. Controversy remains, however, regarding whether MSCs protect the kidney by transforming to renal tubular epithelium $(1,5,6)$. Several previous studies have also demonstrated the presence of $\mathrm{Y}$ chromosome staining of tubular epithelial cells in injured kidney transplants from female donors into male recipients, suggesting that stem cells (SCs) derived from the male bone marrow migrate and differentiate into the epithelial cells $(37,38)$. Morigi et al (5) also demonstrated that the renoprotective effects of BM-derived SC in acute renal failure are not caused by the hematopoietic stem cells but the MSCs. Broekema et al (39) demonstrated that the tubular engraftment of BM-derived cells only occurs dependent on the severity of renal damage following ischemia-reperfusion injury and that, when transplanted, these cells acquire an epithelial phenotype. However, additional previous studies revealed that there was very little or no tubular incorporation, despite exogenous MSCs having a protective effect on kidney injury (40-42). The reason for such discrepancies between these studies is unclear, but may be associated with the degree of severity of the model and the protocols used, the types of stem cells and the labeling methods.

Morigi et al (43) used human cord blood MSC in a mouse model of acute renal failure, revealing that the quantity of stem cells reaching the kidney was very low, and the number of stem cells located in kidney tubules was lower still; most stem cells were located in the renal interstitium. In this previous study, there were $2 \pm 0.4 \times 10^{5}$ stem cells located in the kidney, $5 \pm 5 \%$ of which were located in tubules. A previous study from our group (3) was also in concordance with this result: When VEGF-labeled hMSCs and hMSCs were used to treat AKI, their localizations in the kidney were $1.63 \pm 0.68$ and $1.58 \pm 0.77 \mathrm{hMSCs} /$ section, respectively. In this previous study, most stem cells were located in the renal interstitium, and only a small quantity of stem cells was located in the renal tubules. In the present study, hMSCs were injected into the kidneys of newborn mice, revealing that $10 \pm 2.1 \%$ of labeled stem cells were localized to tubules, and that the proportion of MSCs within the renal tubular epithelium was higher than that in previous studies $(3,43)$. The causes for the discrepancy between this and previous studies may include the following: Firstly, nephron development was not completed within 2 weeks of the birth of mice; secondly, immunogenicity of stem cells from embryo was reduced such that the transplanted stem cells were able to participate in the development of the renal tubule during this period $(44,45)$. In our previous study, it was demonstrated that the number of stem cells recruited in the kidney through intravenous injection was very limited (3). A large number of MSCs were observed in other organs besides the kidney, such as in the liver, lungs and spleen, between $2 \mathrm{~h}$ and 4 days of hMSC infusion. In the current study, numerous competent cells were implanted through a renal local injection approach.

Yokoo et al (46) reported that MSC cells differentiate into kidney structures subsequent to their injection into the rat intermediate mesoderm at the nephrogenic site of the embryo. However, after 6 days of injection of MSC in the developing metanephros, it was revealed that MSC were not implanting into the renal tubules. The authors therefore concluded that MSCs required nephrogenic signals to participate in metanephros development. In the present study, however, MSCs were revealed to be implanted in tubules 2 weeks after the injection, which indicated MSC may differentiate into tubular epithelium during kidney development. The difference in present and previous outcomes may be attributed to different observation times following the injection, different animal models and types of stem cell.

A previous study by Herzog et al (47) reports cell fusion as a mechanism of BM-derived cell epithelial differentiation, and Fang et al (48) demonstrated that among BM-derived cells integrated into the renal epithelium ( $\sim 10 \%$ of the cells), a number revealed signs of fusion to the renal tubular epithelium. The present study did not exclude the fusion of hMSCs and renal tubular epithelium of mice as a potential mechanism, but multiple previous in vitro and in vivo studies suggest that MSCs may express specific molecules and aquaporins of tubular epithelium $(48,49)$.

In conclusion, it was demonstrated that: i) PKH-26 label had no effect on stem cell proliferation and differentiation ability; ii) hMSCs may participate in kidney development through differentiation into renal tubular epithelium when the approach of renal local injection into the kidney of newborn mice was adopted. In the present study, it was therefore concluded that hMSCs may be differentiated into renal tubule epithelium during kidney development.

\section{Acknowledgements}

The present work is supported by the National Natural Science Foundation of China (grant no. 81200490), the Medical Science and Technology Development Foundation of Jiangsu Province Department of Health (grant no. 201213).

\section{References}

1. Morigi M and Benigni A: Mesenchymal stem cells and kidney repair. Nephrol Dial Transplant 28: 788-793.

2. Imai $\mathrm{E}$ and Iwatani $\mathrm{H}$ : The continuing story of renal repair with stem cells. J Am Soc Nephrol 18: 2423-2424, 2007.

3. Yuan L, Wu MJ, Sun HY, Xiong J, Zhang Y, Liu CY, Fu LL, Liu DM, Liu HQ and Mei CL: VEGF-modified human embryonic mesenchymal stem cell implantation enhances protection against cisplatin-induced acute kidney injury. Am J Physiol Renal Physiol 300: F207-F218, 2011.

4. Selby NM, Crowley L, Fluck RJ, Mcintyre CW, Monaghan J, Lawson $\mathrm{N}$ and Kolhe NV: Use of electronic results reporting to diagnose and monitor AKI in hospitalized patients. Clin J Am Soc Nephrol 7: 533-540, 2012.

5. Morigi M, Imberti B, Zoja C, Corna D, Tomasoni S, Abbate M, Rottoli D, Angioletti S, Benigni A, Perico N, et al: Mesenchymal stem cells are renotropic, helping to repair the kidney and improve function in acute renal failure. J Am Soc Nephrol 15: 1794-1804, 2004. 
6. Bussolati B, Hauser PV, Carvalhosa R and Camussi G: Contribution of stem cells to kidney repair. Curr Stem Cell Res Ther 4: $2-8,2009$.

7. Chhabra P and Brayman KL: The use of stem cells in kidney disease. Curr Opin Organ Transplant 14: 72-78, 2009.

8. Yokote S, Yamanaka S and Yokoo T: De novo kidney regeneration with stem cells. J Biomed Biotechnol 2012: 453519, 2012

9. Hwang JH, Shim SS, Seok OS, Lee HY, Woo SK, Kim BH, Song HR, Lee JK and Park YK: Comparison of cytokine expression in mesenchymal stem cells from human placenta, cord blood, and bone marrow. J Korean Med Sci 24: 547-554, 2009.

10. Cao H, Heazlewood SY, Williams B, Cardozo D, Nigro J, Oteiza A and Nilsson SK: The role of CD44 in fetal and adult hematopoietic stem cell regulation. Haematologica 101: 26-37, 2016.

11. Gotherstrom C, Ringden O, Westgren M, Tammik C and Le Blanc K: Immunomodulatory effects of human foetal liver-derived mesenchymal stem cells. Bone Marrow Transplant 32: 265-272, 2003.

12. Li O, Tormin A, Sundberg B, Hyllner J, Le Blanc K and Scheding S: Human embryonic stem cell-derived mesenchymal stroma cells (hES-MSCs) engraft in vivo and support hematopoiesis without suppressing immune function: Implications for off-the shelf ES-MSC therapies. PLoS One 8: e55319, 2013.

13. O'Donoghue K and Fisk NM: Fetal stem cells. Best Pract Res Clin Obstet Gynaecol 18: 853-875, 2004.

14. Le Blanc K: Immunomodulatory effects of fetal and adult mesenchymal stem cells. Cytotherapy 5: 485-489, 2003.

15. Le Blanc K, Tammik L, Sundberg B, Haynesworth SE and Ringden O: Mesenchymal stem cells inhibit and stimulate mixed lymphocyte cultures and mitogenic responses independently of the major histocompatibility complex. Scand J Immunol 57: 11-20, 2003

16. Emanueli C, Lako M, Stojkovic M and Madeddu P: In search of the best candidate for regeneration of ischemic tissues: Are embryonic/fetal stem cells more advantageous than adult counterparts? Thromb Haemost 94: 738-749, 2005.

17. Wu M, Yang L, Liu S, Li H, Hui N, Wang F and Liu $\mathrm{H}$ Differentiation potential of human embryonic mesenchymal stem cells for skin-related tissue. Br J Dermatol 155: 282-291, 2006.

18. Institute of Laboratory Animal Resources (US). Committee on Care, Use of Laboratory Animals, and National Institutes of Health (US). Division of Research Resources: Guide for the care and use of laboratory animals. 8th edition. National Academies Press, Washington, DC, 2011.

19. Schimke MM, Marozin S and Lepperdinger G: Patient-specific age: The other side of the coin in advanced mesenchymal stem cell therapy. Front Physiol 6: 362, 2005.

20. Sterneckert JL, Reinhardt P and Scholer HR: Investigating human disease using stem cell models. Nat Rev Genet 15: 625-639, 2014

21. Allers C, Lasala GP and Minguell JJ: Presence of osteoclast precursor cells during ex vivo expansion of bone marrow-derived mesenchymal stem cells for autologous use in cell therapy. Cytotherapy 16: 454-459, 2013.

22. Semon JA, Maness C, Zhang X, Sharkey SA, Beuttler MM, Shah FS, Pandey AC, Gimble JM, Zhang S, Scruggs BA, et al: Comparison of human adult stem cells from adipose tissue and bone marrow in the treatment of experimental autoimmune encephalomyelitis. Stem Cell Res Ther 5: 2, 2014.

23. Barry FP and Murphy JM: Mesenchymal stem cells: Clinical applications and biological characterization. Int $\mathrm{J}$ Biochem Cell Biol 36: 568-584, 2004

24. Zhang ZY, Teoh SH, Chong MS, Schantz JT, Fisk NM, Choolani MA and Chan J: Superior osteogenic capacity for bone tissue engineering of fetal compared with perinatal and adult mesenchymal stem cells. Stem Cells 27: 126-137, 2009.

25. Kawaguchi K, Katsuyama Y, Kikkawa S, Setsu $T$ and Terashima T: PKH26 is an excellent retrograde and anterograde fluorescent tracer characterized by a small injection site and strong fluorescence emission. Arch Histol Cytol 73: 65-72, 2010.

26. Gallagher SJ, Shank JA, Bochner BS and Wagner EM: Methods to track leukocyte and erythrocyte transit through the bronchial vasculature in sheep. J Immunol Methods 271: 89-97, 2002.

27. Ude CC, Shamsul BS, Ng MH, Chen HC, Norhamdan MY, Aminuddin BS and Ruszymah BH: Bone marrow and adipose stem cells can be tracked with PKH26 until post staining passage 6 in in vitro and in vivo. Tissue Cell 44: 156-163, 2012.
28. Shao-Fang Z,Hong-Tian Z,Zhi-Nian Z and Yuan-Li H: PKH26 as a fluorescent label for live human umbilical mesenchymal stem cells. In Vitro Cell Dev Biol Anim 47: 516-520, 2011.

29. Haas SJ, Bauer P, Rolfs A and Wree A: Immunocytochemical characterization of in vitro $\mathrm{PKH} 26$-labelled and intracerebrally transplanted neonatal cells. Acta Histochem 102: 273-280, 2000

30. Fox IJ, Daley GQ, Goldman SA, Huard J, Kamp TJ and Trucco M: Stem cell therapy. Use of differentiated pluripotent stem cells as replacement therapy for treating disease. Science 345: 1247391, 2014.

31. Pan Q, Qin X, Ma S, Wang H, Cheng K, Song X, Gao H, Wang Q, Tao R, Wang Y, et al: Myocardial protective effect of extracellular superoxide dismutase gene modified bone marrow mesenchymal stromal cells on infarcted mice hearts. Theranostics 4: 475-486, 2014.

32. Rashid ST and Vallier L: Induced pluripotent stem cells-alchemist's tale or clinical reality? Expert Rev Mol Med 12: 25, 2010.

33. Togel F, Hu Z, Weiss K, Isaac J, Lange C and Westenfelder C: Administered mesenchymal stem cells protect against ischemic acute renal failure through differentiation-independent mechanisms. Am J Physiol Renal Physiol 289: F31-F42, 2005.

34. Semedo P, Palasio CG, Oliveira CD, Feitoza CQ, Gonçalves GM, Cenedeze MA, Wang PM, Teixeira VP, Reis MA, Pacheco-Silva A and Câmara NO: Early modulation of inflammation by mesenchymal stem cell after acute kidney injury. Int Immunopharmacol 9: 677-682, 2009.

35. Gatti S, Bruno S, Deregibus MC, Sordi A, Cantaluppi V, Tetta C and Camussi G: Microvesicles derived from human adult mesenchymal stem cells protect against ischaemia-reperfusion-induced acute and chronic kidney injury. Nephrol Dial Transplant 26: 1474-1483, 2011.

36. Bruno S, Grange C, Deregibus MC, Calogero RA, Saviozzi S, Collino F, Morando L, Busca A, Falda M, Bussolati B, et al: Mesenchymal stem cell-derived microvesicles protect against acute tubular injury. J Am Soc Nephrol 20: 1053-1067, 2009.

37. Poulsom R, Forbes SJ, Hodivala-Dilke K, Ryan E, Wyles S, Navaratnarasah S, Jeffery R, Hunt T, Alison M, Cook T, et al: Bone marrow contributes to renal parenchymal turnover and regeneration. J Pathol 195: 229-235, 2001.

38. Gupta S, Verfaillie C, Chmielewski D, Kim Y and Rosenberg ME: A role for extrarenal cells in the regeneration following acute renal failure. Kidney Int 62: 1285-1290, 2002.

39. Broekema M, Harmsen MC, Koerts JA, Petersen AH, van Luyn MJ, Navis G and Popa ER: Determinants of tubular bone marrow-derived cell engraftment after renal ischemia/reperfusion in rats. Kidney Int 68: 2572-2581, 2005.

40. Lin F, Moran A and Igarashi P: Intrarenal cells, not bone marrow-derived cells, are the major source for regeneration in postischemic kidney. J Clin Invest 115: 1756-1764, 2005.

41. Duffield JS, Park KM, Hsiao LL, Kelley VR, Scadden DT, Ichimura T and Bonventre JV: Restoration of tubular epithelial cells during repair of the postischemic kidney occurs independently of bone marrow-derived stem cells. J Clin Invest 115: 1743-1755, 2005.

42. Duffield JS and Bonventre JV: Kidney tubular epithelium is restored without replacement with bone marrow-derived cells during repair after ischemic injury. Kidney Int 68: 1956-1961, 2005.

43. Morigi M, Rota C, Montemurro T, Montelatici E, Lo Cicero V, Imberti B, Abbate M, Zoja C, Cassis P, Longaretti L, et al: Life-sparing effect of human cord blood-mesenchymal stem cells in experimental acute kidney injury. Stem Cells 28: 513-522, 2010.

44. Sorokin L and Ekblom P: Development of tubular and glomerular cells of the kidney. Kidney Int 41: 657-664, 1992.

45. Narbaitz R, Vandorpe D and Levine DZ: Differentiation of renal intercalated cells in fetal and postnatal rats. Anat Embryol (Berl) 183: 353-361, 1991

46. Yokoo T, Ohashi T, Shen JS, Sakurai K, Miyazaki Y, Utsunomiya Y, Takahashi M, Terada Y, Eto Y, Kawamura T, et al: Human mesenchymal stem cells in rodent whole-embryo culture are reprogrammed to contribute to kidney tissues. Proc Natl Acad Sci USA 102: 3296-3300, 2005.

47. Herzog EL and Krause DS: Engraftment of marrow-derived epithelial cells: The role of fusion. Proc Am Thorac Soc 3: 691-695, 2006.

48. Fang TC, Alison MR, Cook HT, Jeffery R, Wright NA and Poulsom R: Proliferation of bone marrow-derived cells contributes to regeneration after folic acid-induced acute tubular injury. J Am Soc Nephrol 16: 1723-1732, 2005.

49. Yadav N, Rao S, Bhowmik DM and Mukhopadhyay A: Bone marrow cells contribute to tubular epithelium regeneration following acute kidney injury induced by mercuric chloride. Indian J Med Res 136: 211-220, 2012 